\title{
Diagnostic importance of pulmonary interleukin-1 $\beta$ and interleukin-8 in ventilator-associated pneumonia
}

\author{
Andrew Conway Morris, ${ }^{1}$ Kallirroi Kefala, ${ }^{1}$ Thomas S Wilkinson, ${ }^{1}$ \\ Olga Lucia Moncayo-Nieto, ${ }^{1}$ Kevin Dhaliwal, ${ }^{1}$ Lesley Farrell, ${ }^{1}$ Timothy S Walsh, ${ }^{2}$ \\ Simon J Mackenzie, ${ }^{2}$ David G Swann, ${ }^{2}$ Peter JD Andrews, ${ }^{3}$ Niall Anderson, ${ }^{4}$ \\ John RW Govan, ${ }^{5}$ lan F Laurenson, ${ }^{6}$ Hamish Reid, ${ }^{7}$ Donald J Davidson, ${ }^{1}$ \\ Christopher Haslett, ${ }^{1}$ Jean-Michel Sallenave, ${ }^{8}$ A John Simpson ${ }^{1}$
}

\section{See Editorial, p190}

- Supplementary tables are published online only at http:// thx.bmj.com/content/vol65/ issue3.

${ }^{1}$ MRC Centre for Inflammation Research, University of Edinburgh, Scotland, UK ${ }^{2}$ Intensive Care Unit, Royal Infirmary of Edinburgh, Scotland, UK ${ }^{3}$ Intensive Care Unit, Western General Hospital, Edinburgh, Scotland, UK ${ }^{4}$ Department of Public Health Sciences, University of Edinburgh, Scotland, UK ${ }^{5}$ Centre for Infectious Diseases, University of Edinburgh, Scotland, UK ${ }^{6}$ Department of Clinical Microbiology, Royal Infirmary of Edinburgh, Scotland, UK ${ }^{7}$ Penicuik Health Centre, Penicuik, Scotland, UK ${ }^{8}$ Institut Pasteur, Unité de Défense Innée et Inflammation, Paris, France

\section{Correspondence to} Dr Andrew Conway Morris, MRC Centre for Inflammation Research, Room C2.17, University of Edinburgh, Edinburgh EH16 4TJ, UK; mozza@doctors.org.uk

Received 23 June 2009 Accepted 11 September 2009

\begin{abstract}
Background Ventilator-associated pneumonia (VAP) is the most commonly fatal nosocomial infection. Clinical diagnosis of VAP remains notoriously inaccurate. The hypothesis was tested that significantly augmented inflammatory markers distinguish VAP from conditions closely mimicking VAP.
\end{abstract}

Methods A prospective, observational cohort study was carried out in two university hospital intensive care units recruiting 73 patients with clinically suspected VAP, and a semi-urban primary care practice recruiting a reference group of 21 age- and sex-matched volunteers. Growth of pathogens at $>10^{4}$ colony-forming units $(\mathrm{cfu}) / \mathrm{ml}$ of bronchoalveolar lavage fluid (BALF) distinguished VAP from "non-VAP". Inflammatory mediators were quantified in BALF and serum. Mediators showing significant differences between patients with and without VAP were analysed for diagnostic utility by receiver operator characteristic (ROC) curves.

Results Seventy-two patients had recoverable lavage $-24 \%$ had VAP. BALF interleukin-1 $\beta$ (IL-1 $\beta)$, IL-8, granulocyte colony-stimulating factor and macrophage inflammatory protein-1 $\alpha$ were significantly higher in the VAP group (all $p<0.005$ ). Using a cut-off of $10 \mathrm{pg} / \mathrm{ml}$, BALF IL-1 $\beta$ generated negative likelihood ratios for VAP of 0.09. In patients with BALF IL- $1 \beta<10 \mathrm{pg} / \mathrm{ml}$ the posttest probability of VAP was $2.8 \%$. Using a cut-off value for IL-8 of $2 \mathrm{ng} / \mathrm{ml}$, the positive likelihood ratio was 5.03 . There was no difference in cytokine levels between patients with sterile BALF and those with growth of $<10^{4} \mathrm{cfu} / \mathrm{ml}$.

Conclusions BALF IL-1 $\beta$ and IL-8 are amongst the strongest markers yet identified for accurately demarcating VAP within the larger population of patients with suspected VAP. These findings have potential implications for reduction in unnecessary antibiotic use but require further validation in larger populations.

\section{INTRODUCTION}

Ventilator-associated pneumonia (VAP) occurs in up to $20 \%$ of patients mechanically ventilated for $>48 \mathrm{~h}$ and is typically associated with mortality rates of $\sim 30 \% .{ }^{1}{ }^{2}$ VAP therefore has a higher mortality than any other hospital-acquired infection, and exerts a large financial burden on health services. ${ }^{3}$ Although estimates of the attributable mortality vary, most studies describe a significant associated mortality and morbidity. ${ }^{13} 4$
The optimal methods and criteria required to make a diagnosis of VAP remain contentious. ${ }^{1}$ However, it is generally accepted that only approximately a quarter to one-third of patients with clinically suspected VAP satisfy predefined microbiological criteria for pneumonia. ${ }^{5}$ The clinical diagnosis of VAP is made relatively frequently in the intensive care unit (ICU). This can result in empirical antibiotic treatment, with the inherent risk of overprescribing. ${ }^{7-9}$ Therefore, in recent years attempts have been made to identify biological markers that can distinguish VAP accurately, rapidly and practically. ${ }^{10-12}$

Given the key roles played by inflammatory cytokines and neutrophils in the natural history of pneumonia, it is plausible that discriminatory markers of infection may be found in the innate immune system. We hypothesised that in critically ill patients pneumonia would drive a further increase in pulmonary inflammation, with inflammatory mediators distinguishing VAP from conditions that mimic VAP.

\section{MATERIALS AND METHODS \\ Patients}

This prospective, observational, cohort study was performed in two university hospital general ICUs admitting all patients except those undergoing cardiothoracic surgery. Patients were screened daily for possible VAP, and were assessed for enrolment as soon as the clinical suspicion was raised. Where enrolment occurred, bronchoscopy took place within $6 \mathrm{~h}$ of clinical diagnosis. Demographic details, co-morbidities and prescribed medications were recorded. The severity of presenting illness was assessed by an Acute Physiology and Chronic Health Evaluation II (APACHE II) score calculated within 24 h of ICU admission. Patients were eligible if they fulfilled recognised criteria for clinically suspected VAP - that is, mechanical ventilation for at least $48 \mathrm{~h}$, new and persisting infiltrates on a chest radiograph and at least two of the following: purulent tracheal secretions, temperature $>38^{\circ} \mathrm{C}$ or white cell count $>11 \times 10^{9} / 1$ - based on a modification of previously published clinical criteria. ${ }^{1}$ Exclusion criteria comprised $\mathrm{PaO}_{2}<8 \mathrm{kPa}$ on $\mathrm{FiO}_{2}$ $>0.7$, positive end-expiratory pressure $>15 \mathrm{~cm}$ $\mathrm{H}_{2} \mathrm{O}$, active bronchospasm, myocardial infarction within the last 3 months, unstable arrhythmia, mean arterial pressure $<65 \mathrm{~mm} \mathrm{Hg}$ on vasopressor online under the BMJ Journals unlocked scheme, see http:// thorax.bmj.com/site/about/ unlocked.xhtml. 
therapy, bleeding diathesis (including platelet count $<20 \times 10^{9} / 1$ ) and initiation or modification of antibiotics in the preceding 3 days. ${ }^{1}$ Patients who had had no change in prescribed antibiotics for $>3$ days were included. ${ }^{1} 13$

Patients had fibreoptic bronchoscopy and bronchoalveolar lavage (BAL) performed by a single experienced operator using a predefined, standardised technique. ${ }^{14} 15$ Briefly, where focal infiltrates were present, the bronchoscope was wedged in a subsegment corresponding to the area of radiological involvement. In the case of diffuse radiographic change the bronchoscope was wedged in a subsegment producing visible purulent secretions or (in the absence of purulent secretions) in the posterior segment of the right lower lobe. A $20 \mathrm{ml}$ aliquot of sterile saline was instilled and the aspirate (representing a "bronchiolar" sample) discarded, then $200 \mathrm{ml}$ of sterile saline was instilled in aliquots and the aspirate (representing an alveolar sample) retained. Whole blood was collected into $0.38 \%$ sodium citrate (final concentration).

\section{Volunteer reference group}

After recruitment of 40 patients the (anonymised) age and sex of each patient was communicated to a local primary care practice, where staff unconnected with the study randomly identified matching individuals and sent out invitations to participate. The first twenty-one respondents were enrolled to form a reference group. Exclusion criteria comprised hypoxia $\left(\mathrm{SaO}_{2}<92 \%\right.$ on air), bleeding diathesis, anticoagulant therapy, insulin-dependent diabetes mellitus, arrhythmia, bronchospasm not responding to nebulised $\beta 2$ agonist or clinical evidence of respiratory tract infection. Eligible volunteers provided blood and had fibreoptic bronchoscopy and BAL performed by the same investigator as above.

\section{Processing of BAL fluid (BALF) and whole blood}

An aliquot of BALF was sent to the National Health Service (NHS) Clinical Microbiology laboratory for culture, whilst simultaneous cultures were undertaken in our research laboratory. Samples were processed using a standard operating procedure (SOP) in accordance with the SOP for the processing of BAL issued by the UK Health Protection Agency (HPA). ${ }^{16}$ Analyses were limited to those routinely performed on BALF in our NHS laboratory (ie, detailed analysis for viruses was not included), with the exception that we additionally performed anaerobic cultures. BALF from healthy volunteers was only cultured in the research laboratory.

Growth of $>10^{4}$ colony-forming units (cfu) per $\mathrm{ml}$ of lavage fluid confirmed VAP. ${ }^{1}$ This definition is used by several infection control and critical care organisations including the Hospitals in Europe Link for Infection Control through Surveillance (HELICS) programme. ${ }^{17}$ Patients whose BALF grew $<10^{4} \mathrm{cfu} / \mathrm{ml}$ formed a "non-VAP" category. For further subgroup analysis "non-VAP" was subdivided into "sub-VAP growth" and "sterile" groups. A $1 \mathrm{ml}$ aliquot of BALF was collected for culture, and the remainder centrifuged at $700 \mathrm{~g}$ for $10 \mathrm{~min}$. Supernatant was immediately frozen at $-80^{\circ} \mathrm{C}$ until further analysis. The cellular pellet was resuspended in warmed Iscove's modified Dulbecco's medium (IMDM; Invitrogen, Carlsbad, California, USA) and cytospins produced. Cytospins were stained with Diff-Quik (Reagena, Toivala, Finland) and differential cell counts established.

A $30 \mathrm{ml}$ aliquot of citrated whole blood was separated into cellular and plasma components by centrifugation. ${ }^{18}$ Serum was prepared by adding $1 \mathrm{M}$ calcium chloride to plasma.

\section{Quantification of cytokines and inflammatory mediators}

Concentrations of tumour necrosis factor- $\alpha$ (TNF $\alpha$ ), interleukin (IL)-1 $\beta$, IL-6, IL-8, IL-10, granulocyte colony-stimulating factor
(G-CSF) and macrophage inflammatory protein-1 $\alpha$ (MIP-1 $1 \alpha)$ in serum and BALF were estimated using cytometric bead array (CBA) kits (BD Bioscience, Franklin Lakes, New Jersey, USA). The concentrations of type 1 soluble triggering receptor expressed on myeloid cells (sTREM-1) and monocyte chemoattractant peptide 1 (MCP-1) were measured by ELISA (R\&D Systems, Minneapolis, Minnesota, USA). Samples measured by CBA and ELISA were diluted in an assay-dependent manner to ensure they lay within the limits of the calibration curves. The dilution required ranged from neat to $1: 100$ for the highest values. Urea was measured by a colorimetric method (QuantiChrom, Bioassay Systems, Hayward, California, USA) and specifically used as a recognised means of correcting for dilutional effects in BALF. ${ }^{19}$

\section{Consent and ethics approval}

Informed, witnessed assent was obtained from a relative or main carer for all patients. Informed, written consent was obtained from all volunteers. The study was approved by the relevant Research Ethics Committees.

\section{Statistical analysis}

Statistical analysis was conducted using Prism (Graphpad Software, San Diego, California, USA). Non-normally distributed data were analysed using the Mann-Whitney $U$ test for two variables and the Kruskal-Wallis test for greater than two variables, using the Dunn method for posthoc analysis. Normally distributed data were analysed using the Student test or analysis of variance (ANOVA) with the Bonferroni method for posthoc analysis. Preliminary identification of candidate biomarkers was undertaken by noting those with significant differences between the VAP and "non-VAP" median values. The diagnostic utility of these variables was assessed using area under the receiver operator characteristic (ROC) curves. For those with area under the curve values of $\geq 0.5$, optimal cut-offs and likelihood ratios were determined by the value with the maximum Youden index ${ }^{20}$; a likelihood ratio is a likelihood that a person with a positive (or negative) test has the disease in question. For the two most promising candidates discriminating VAP from "non-VAP", multilevel likelihood ratios were calculated to illustrate diagnostic potential. Combinations of measures were assessed for enhanced diagnostic potential by statistical modelling via logistic regression and classification tree methods.

\section{RESULTS}

There were 74 eligible patients; 73 were enrolled, with one excluded due to lack of a relative's informed assent. Seventy-two patients had recoverable BALF and so entered the analysis. Seventeen (24\%) grew organisms at $>10^{4} \mathrm{cfu} / \mathrm{ml}$ of BALF ("VAP group") (growing a median of $5.7 \times 10^{4} \mathrm{cfu} / \mathrm{ml}$, IOR $3 \times 10^{4}-6 \times 10^{4} \mathrm{cfu} / \mathrm{ml}$ ). Seven grew a Gram-positive organism, six a Gram-negative organism, and four patients grew fungi, including three yeasts (table 1). One patient growing a Grampositive organism also grew anaerobes. The remaining 55 patients formed the "non-VAP" group, of whom 22 were categorised as "sub-VAP" (ie, organisms cultured but at $<10^{4} \mathrm{cfu} / \mathrm{ml}$ of BALF, growing a median $2.1 \times 10^{2} \mathrm{cfu} / \mathrm{ml}\left(\mathrm{IOR} 7.5 \times 10^{2}-4 \times 10^{2} \mathrm{cfu} / \mathrm{ml}\right)$ ) and 33 as "sterile". Comparisons between cultures conducted in the NHS laboratory and our laboratory revealed strong agreement, with all patients identified as having VAP by the research laboratory being similarly identified by the NHS laboratory. There were no discordant cultures in this group. Amongst the non-VAP group two patients grew bacteria $\left(<10^{2} \mathrm{cfu} / \mathrm{ml}\right)$ which were not reported by the NHS laboratory, and one sample grew 
Table 1 Organisms grown in patients with and without ventilatorassociated pneumonia (VAP)

\begin{tabular}{lll}
\hline Organisms & VAP & Non-VAP \\
\hline Streptococcus pneumoniae & 0 & 1 \\
Methicillin-sensitive Staphylococcus & 2 & 2 \\
aureus & & \\
Methicillin-resistant S aureus & 3 & 6 \\
Coagulase-negative Staphylococcus spp. & 2 & 1 \\
Haemophilus influenzae & 1 & 2 \\
Escherichia coli & 3 & 1 \\
Citrobacter freundii & 1 & 0 \\
Citrobacter koseri & 0 & 1 \\
Enterobacter cloacae & 1 & 2 \\
Acinetobacter baumannii & 0 & 1 \\
Pseudomonas aeruginosa & 0 & 2 \\
Serratia marcescens & 0 & 1 \\
Klebsiella pneumoniae & 0 & 4 \\
Candida albicans & 3 & 2 \\
Aspergillus fumigatus & 1 & 1 \\
Anaerobes & 1 & 0
\end{tabular}

bacteria $\left(<10^{2} \mathrm{cfu} / \mathrm{ml}\right)$ in the NHS laboratory but not the research laboratory. Twenty-one volunteers were recruited to form the reference group. Twelve samples grew mixed oral commensal flora but always at $<10^{2} \mathrm{cfu} / \mathrm{ml}$.

In the VAP group 18 species were grown from 17 patients (ie, two organisms were grown from one patient); by definition organisms were grown at $>10^{4} \mathrm{cfu} / \mathrm{ml}$ BALF. In the non-VAP group 27 species were grown from 22 patients (ie, two organisms were grown from five patients); by definition organisms were grown at $<10^{4} \mathrm{cfu} / \mathrm{ml}$ BALF.

The three groups studied were closely matched with respect to age (table 2). The VAP and non-VAP groups were similar with regard to duration of mechanical ventilation, severity of illness and co-morbidities (table 2). Although there was a greater likelihood of patients in the VAP group being male, having a surgical reason for admission to ICU and having less acute lung injury (ALI)/adult respiratory distress syndrome (ARDS), these differences did not reach statistical significance (table 2).

All measured cytokines and inflammatory mediators in serum showed similar concentrations in the VAP and non-VAP groups (Supplementary table S1). No serum marker appeared to have potential value for discriminating VAP from non-VAP, though a trend in this direction was observed for sTREM-1. In general, serum markers were higher in both patient groups than in volunteers, with the exceptions of IL-1 $\beta$ and TNF $\alpha$, which were broadly similar in all three groups.
In contrast, the VAP group had significantly higher concentrations of IL-1 $\beta$, IL-8, G-CSF and MIP-1 $\alpha$ in BALF than the nonVAP group (table 3 ). Trends in the same direction were observed for IL-6 and sTREM-1 (table 3).

On the basis of these findings, the capacity for IL-1 $\beta$, IL-8, GCSF, MIP- $1 \alpha$, IL- 6 and sTREM- 1 to distinguish VAP among the population with clinically suspected VAP was tested (figure 1). For the ROC curves plotted in figure 1, area under the curve correlates with the discriminatory value of the marker being analysed. In this context IL-1 $\beta$ and IL-8 appeared to delineate VAP most accurately. When optimal cut-off values were derived, concentrations of BALF IL- $1 \beta<10 \mathrm{pg} / \mathrm{ml}$ appeared to be particularly powerful for the exclusion of VAP. Specifically, where BALF IL-1 $\beta$ was $<10 \mathrm{pg} / \mathrm{ml}$, the negative likelihood ratio of 0.09 gives a post-test probability of having VAP calculated at $2.8 \%(95 \% \mathrm{CI}$ $0.1 \%$ to $15.9 \%$ ). IL-1 $\beta$ proved less useful as a positive discriminator of VAP. In contrast, IL-8, whilst being less powerful for the exclusion of VAP, had a broader diagnostic value. Thus, relative to the identified cut-off value in figure 1 of $2000 \mathrm{pg} / \mathrm{ml}$, a high BALF IL-8 concentration increased the post-test probability of VAP being present to $61.4 \%$ (95\% CI $36.2 \%$ to $76.8 \%$ ), derived from the positive likelihood ratio of 5.03. These data are presented as scatter plots in figure 2, illustrating that IL-1 $\beta$ has utility in excluding VAP, but yields a number of false positives. In contrast, IL-8 has a broader utility, but demonstrates a number of falsepositive and false-negative values. Multilevel likelihood ratios demonstrated that increasing levels of BALF IL-8 lead to increased confidence for "ruling in" VAP, with the highest level examined $(4000 \mathrm{ng} / \mathrm{ml})$ producing a post-test probability of $75 \%$. However, at this level, patient numbers are small and there is a marked reduction in sensitivity (details are presented in Supplementary table S2). A similar relationship was not demonstrated for IL1- $\beta$ (see table S2). G-CSF, MIP-1 $\alpha$, IL- 6 and sTREM-1 had markedly less discriminatory value than IL- $1 \beta$ or IL-8. Statistical modelling using combinations of mediators (via logistic regression and classification tree methods) failed to add discriminatory value to that achieved by either IL-1 $\beta$ or IL-8 alone. We did not find any difference in cytokine levels between VAPs caused by different classes of organism (Gram positive, Gram negative or fungi). Interestingly all cases associated with Candida albicans had cytokine levels above the optimum cut-off point for diagnosis for IL-1 $\beta$, IL-8, G-CSF and MIP- $1 \alpha$.

To examine the specificity of our findings, we analysed the cytokine levels in the non-VAP group subdivided into those with no growth ("sterile") and those with growth below the $10^{4} \mathrm{cfu} / \mathrm{ml}$ cut off ("sub-VAP growth"). Cytokine concentrations did not differ significantly between the sub-VAP and sterile groups (figure 3). No correlation was found between bacterial growth

Table 2 Demographic and clinical details of patients and age-/sex-matched volunteers

\begin{tabular}{llll}
\hline & VAP (n=17) & Non-VAP (n=55) & \multicolumn{1}{c}{$\begin{array}{l}\text { Matched } \\
\text { volunteers (n=21) }\end{array}$} \\
\hline Mean age (range) & $57(31-83)$ & $58(25-87)$ & $59(24-84)$ \\
\%Male & $76 \%$ & $55 \%$ & $79 \%$ \\
Mean (95\% CI) APACHE II score & $23(20-26)$ & $21(20-23)$ & NA \\
Median (IOR) days of ventilation before enrolment & $8(6-9)$ & $8(5-10)$ & NA \\
ICU mortality & $35 \%$ & $36 \%$ & NA \\
\% With surgical diagnosis on admission & $65 \%$ & $47 \%$ & NA \\
\% With $\geq 1$ co-morbidity & $59 \%$ & $56 \%$ & NA \\
\% Receiving immunosuppressant drugs (including corticosteroids) & $12 \%$ & $11 \%$ & NA \\
\% Receiving antibiotics on day of diagnosis & $29 \%$ & $60 \%$ & NA \\
\% With acute lung injury/acute respiratory distress syndrome & $17 \%$ & $35 \%$ & NA \\
\% With systemic inflammatory response syndrome (SIRS) & $88 \%$ & $81 \%$ & \\
\hline
\end{tabular}


A

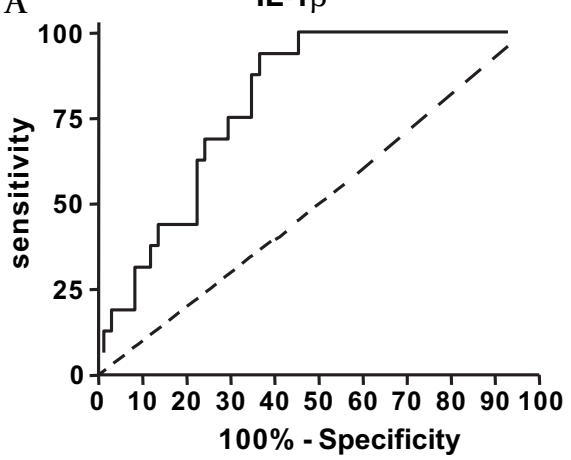

C

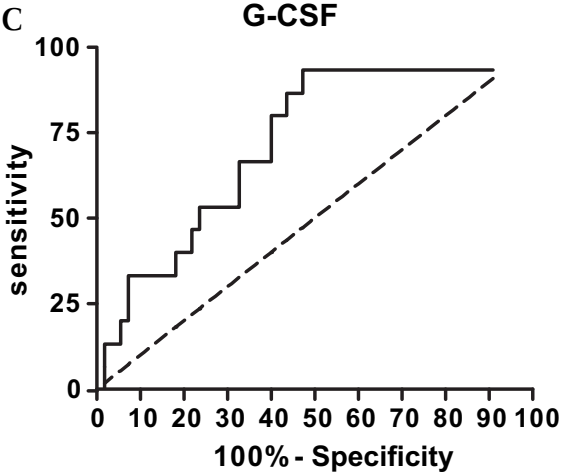

IL-6

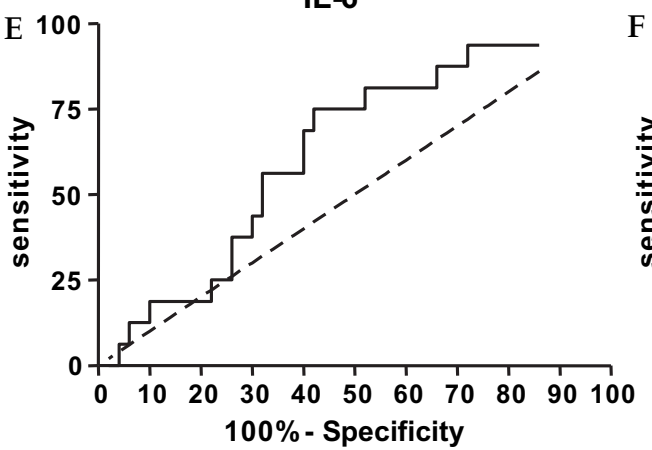

B
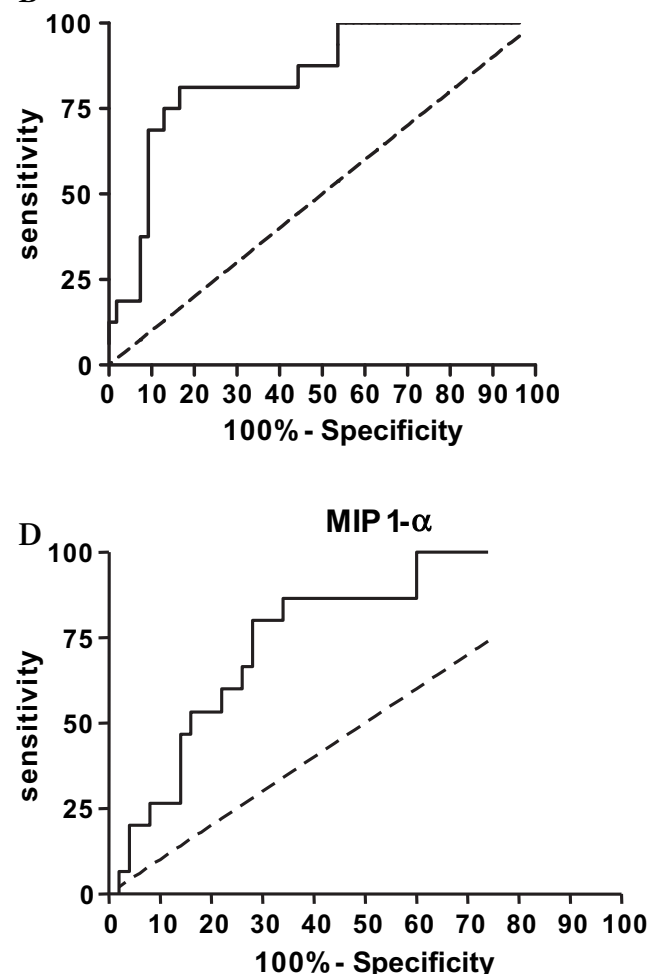

STREM-1

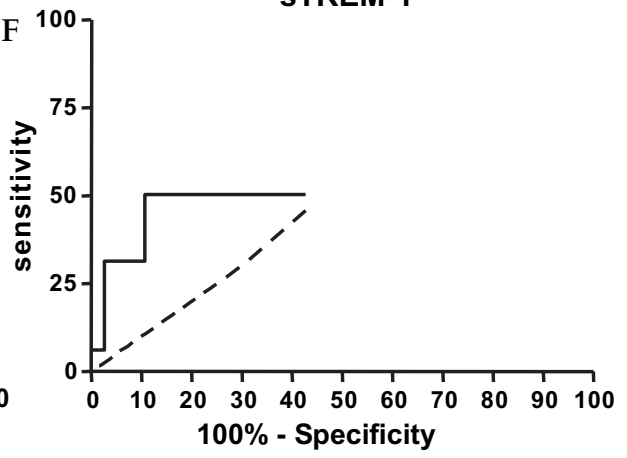

\begin{tabular}{|c|c|c|c|c|c|c|c|c|}
\hline Cytokine & 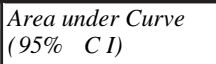 & $\begin{array}{l}\text { Optimal cut-off } \\
(\mathrm{pg} / \mathrm{ml})\end{array}$ & Sensitivity & Specificity & $P P V$ & $N P V$ & $+L R$ & $-L R$ \\
\hline$\overline{\mathrm{IL}-1 \S}$ & $\begin{array}{|ll|}0.81 & \\
(0.71 & -0.91)\end{array}$ & 10 & $94 \%$ & $64 \%$ & $43 \%$ & $97 \%$ & 2.46 & 0.09 \\
\hline IL - 8 & $\begin{array}{ll}0.83 & \\
(0.74 & -0.95)\end{array}$ & 2000 & $81 \%$ & $83 \%$ & $59 \%$ & $94 \%$ & 5.03 & 0.20 \\
\hline G-CS F & $0.73(0.58-0.86)$ & 37 & $80 \%$ & $60 \%$ & $35 \%$ & $92 \%$ & 2.0 & 0.33 \\
\hline MIP $-1 \alpha$ & $0.773(0.66-0.9)$ & 14 & $80 \%$ & $72 \%$ & $46 \%$ & $92 \%$ & 2.9 & 0.27 \\
\hline IL -6 & $0.63(0.48-0.78)$ & \multicolumn{7}{|c|}{ Area under ROC curve does not differ significantly from identity } \\
\hline STREM -1 & $0.66(0.48-0.83)$ & \multicolumn{7}{|c|}{ Area under ROC curve does not differ significantly from identity } \\
\hline
\end{tabular}

Figure 1 Receiver operating characteristic (ROC) curves and optimal sensitivity, specificity, positive predictive values (PPVs) and negative predictive values (NPVs) for bronchoalveolar lavage fluid (BALF) cytokines ( $n=72,55$ non-ventilator-associated pneumonia (VAP) and 17 VAP). Data are derived from the patients with clinically suspected VAP. The broken line shows identity. G-CSF, granulocyte colony-stimulating factor; IL, interleukin; +LR, positive likelihood ratio; -LR, negative likelihood ratio; MIP 1- $\alpha$, macrophage inflammatory protein-1 $\alpha$; sTREM-1; type 1 soluble triggering receptor expressed on myeloid cells. 
Table 3 Inflammatory profile of bronchoalveolar lavage fluid (BALF)

\begin{tabular}{|c|c|c|c|c|}
\hline & $\operatorname{VAP}(n=17)$ & Non-VAP $(n=55)$ & $\begin{array}{l}\text { Matched } \\
\text { volunteers }(n=21)\end{array}$ & $\begin{array}{l}\text { p Value (Dunn } \\
\text { posthoc test } \\
\text { between VAP } \\
\text { and non-VAP) }\end{array}$ \\
\hline Neutrophils $\left(10^{8} / I\right)^{* * *}$ & $2.8(0.6-21)$ & $3.2(0.3-12)$ & $0(0-0.03)$ & NS \\
\hline Macrophages $\left(10^{8} / I\right)^{*}$ & $2.3(0.4-3.9)$ & $1.6(0.2-3.9)$ & $2.9(1.4-3.9)$ & NS \\
\hline IL-1 $\beta(p g / m l)^{* * *}$ & $103(27-755)$ & $3(0-48)$ & $0(0-1.6)$ & $<0.001$ \\
\hline IL-8 (pg/ml) ${ }^{* * *}$ & $6773(2633-11762)$ & $230(68-1072)$ & $69(26-374)$ & $<0.001$ \\
\hline $\mathrm{G}-\mathrm{CSF}(\mathrm{pg} / \mathrm{ml})^{* *}$ & $107(38-383)$ & $17(5-89)$ & $6(0-25)$ & $<0.05$ \\
\hline IL-10 (pg/ml)* & $0(0-9)$ & $1(0-16)$ & $0(0-0)$ & NS \\
\hline sTREM-1 (pg/ml) & $13(0-530)$ & $0(0-7)$ & $0(0-93)$ & NS \\
\hline MIP-1 $1 \alpha(p g / m l)^{* * *}$ & $51(14-269)$ & $3(0-27)$ & $2(0-13)$ & $<0.01$ \\
\hline MCP-1 $(\mathrm{pg} / \mathrm{ml})^{* *}$ & $293(94-554)$ & $173(49-1097)$ & $21(0-54)$ & NS \\
\hline
\end{tabular}

Data are expressed as median and IOR. BALF values are corrected for dilution against concentrations of urea. ${ }^{19}$ Analysis by Kruskall-Wallis analysis of variance (ANOVA) allows detection of any difference between the three groups $\left({ }^{*}<0.05 .{ }^{* *}<0.01,{ }^{* *}<0.001\right.$ shown in the left-hand column). Posthoc comparison between VAP and non-VAP groups was undertaken by Dunn posthoc test, with the $\mathrm{p}$ value displayed in the right-hand column.

G-CSF, granulocyte colony-stimulating factor-1; IL, interleukin; MCP-1, monocyte chemoattractant peptide 1; MIP-1 $\alpha$; macrophage inflammatory protein-1 $\alpha$; NS, non-significant; sTREM-1, type 1 soluble triggering receptor expressed on myeloid cells; TNF $\alpha$, tumour necrosis factor $\alpha$; VAP, ventilator-associated pneumonia.

and cytokine concentration when examining those patients with growth below the $10^{4} \mathrm{cfu} / \mathrm{ml}$ cut-off $(\mathrm{r}=0.14$, $\mathrm{p}=0.31$ for IL-8; $r=0.06, p=0.65$ for IL- $1 \beta$ by Spearman rho).

\section{DISCUSSION}

These data have implications both for the diagnosis of VAP and for understanding the biology of the disease. The practical implications are that, in our hands, a patient with a BALF IL-1 $\beta$ concentration $<10 \mathrm{pg} / \mathrm{ml}$ has an $\sim 3 \%$ probability of having VAP. In contrast, the probability of VAP being present increases as the BALF IL-8 concentration increases; a level $>2 \mathrm{ng} / \mathrm{ml}$ corresponding to a $61 \%$ probability of VAP being present.

VAP remains common and associated with a high mortality. ${ }^{14}$ It is difficult to diagnose accurately on clinical grounds alone, ${ }^{5} 78$ with a tendency to overdiagnosis leading to overprescription of antibiotics. ${ }^{178}$ Diagnostic confirmation remains largely reliant on standard microbiological culture techniques which generally take $24-48 \mathrm{~h}$ to yield results. ${ }^{15}$ In this regard, the capacity of IL-8 to increase the likelihood of a correct diagnosis fivefold and/or the capacity of low BALF IL-1 $\beta$ to reduce the likelihood of VAP 10fold is an important development. The assays used here can yield a result within $4 \mathrm{~h}$. These tests could therefore have a significant impact on clinical decision making.

These results also provide an interesting perspective on the biology of VAP. For the majority of markers both patient groups had higher levels than volunteers, implying a proinflammatory state in critically ill patients as might be expected. However, it is intriguing that acute inflammatory cytokines such as IL-1 $\beta$ and MIP- $1 \alpha$ were so similar when comparing the non-VAP group with volunteers. Although non-significant, there was a trend towards more cases of ARDS/ALI in the non-VAP group. One might have anticipated that these (ARDS/ALI) patients would also demonstrate elevated cytokine levels ${ }^{21}$; however, we found no difference between IL- 8 and IL-1 $\beta$ levels within the non-VAP
Figure 2 Scatter plots of pulmonary cytokine levels $(n=72,55$ nonventilator-associated pneumonia (VAP) and 17 VAP). Each dot represents a single observation. The solid lines mark the median values; the hashed line marks the optimal diagnostic cut-off. A log scale is used due to the skewed nature of cytokine levels. G-CSF, granulocyte colony-stimulating factor; IL, interleukin; MIP1- $\alpha$, macrophage inflammatory protein- $1 \alpha$;
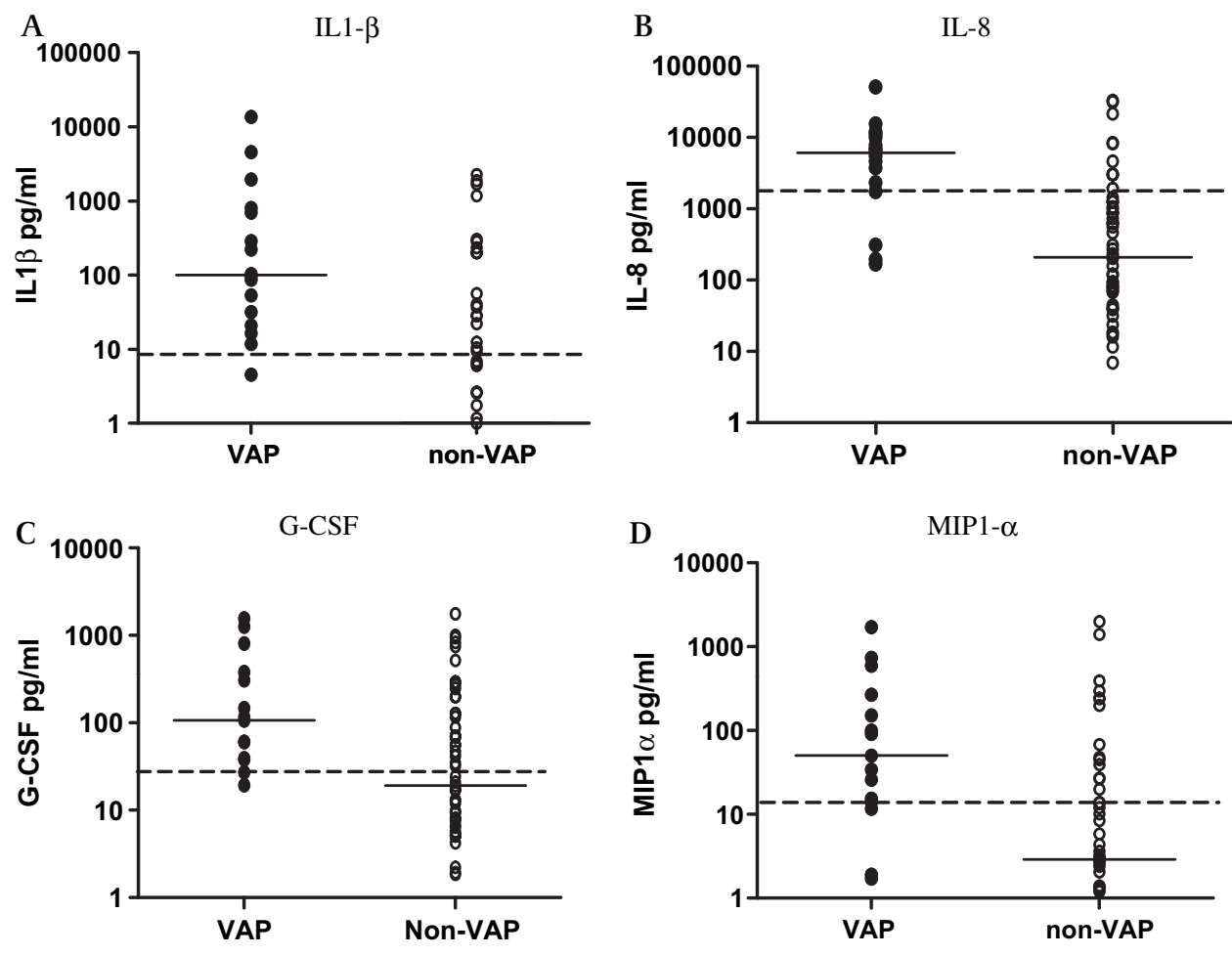
A

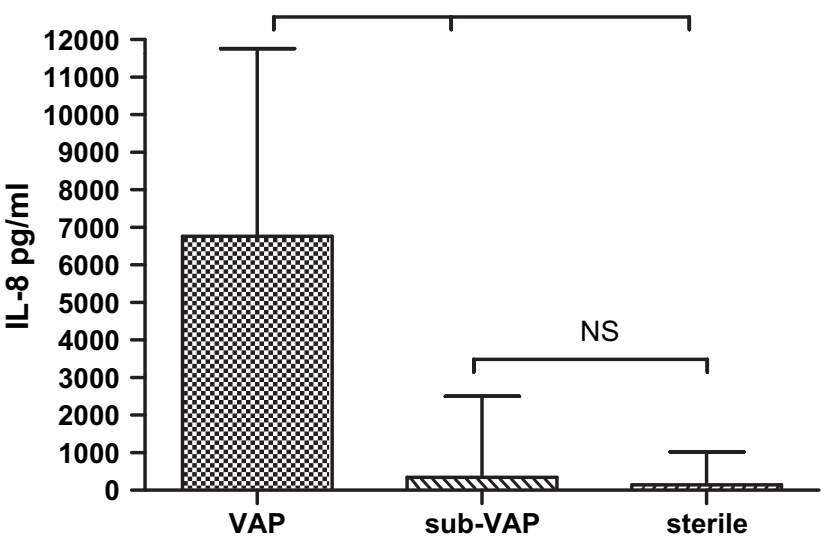

B

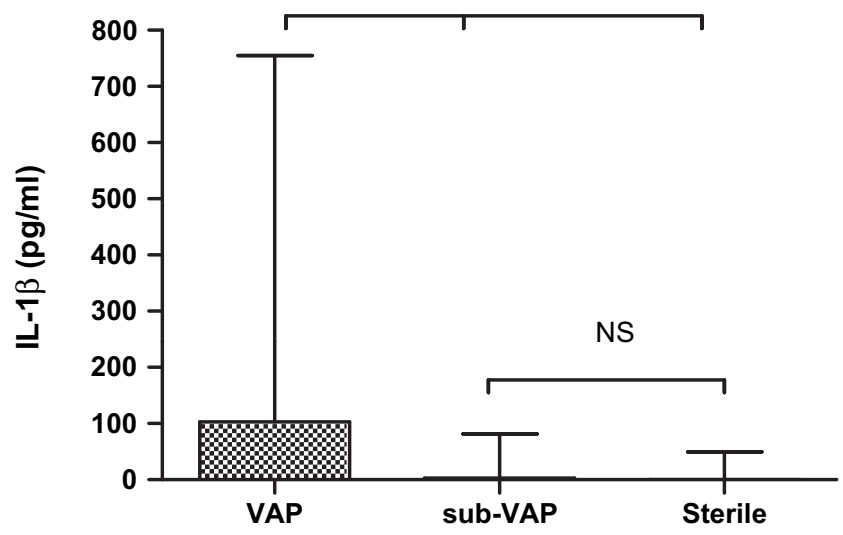

Figure 3 Comparison of pulmonary cytokine levels between patients with ventilator-associated pneumonia (VAP) $(n=17)$, patients with growth of pathogens in bronchoalveolar lavage fluid (BALF) below the diagnostic $10^{4} \mathrm{cfu} / \mathrm{ml}$ cut-off $(\mathrm{n}=22)$, and patients with no growth in BALF $(n=33)$. (A) Interleukin-8 (IL-8) levels. Data are presented as the median and IQRs, $\mathrm{p}<0.0001$ by Kruskal-Wallis; NS (non-significant), $\mathrm{p}>0.05,{ }^{* *} \mathrm{p}<0.01,{ }^{* * *} \mathrm{p}<0.0001$ by the Dunn posthoc test. (B) IL-1 $\beta$ levels. Data are presented as the median and IQRs, $p=0.0006$ by Kruskal-Wallis; NS, $p>0.05,{ }^{* *} p<0.01,{ }^{* *} p<0.0001$ by the Dunn posthoc test.

group when subdivided into those with ARDS/ALI and those without. We believe this relates to the time point of sampling, with our patients' median length of stay prior to being recruited being 8 days.

In contrast, the VAP group appeared to have a brisk inflammatory response confined to the lung. We have no data relating to the source of the relevant cytokines, but diffusion from serum seems unlikely, suggesting a prominent role for alveolar epithelium and/or lung macrophages. It is tempting to speculate that sufficient pathogens in the alveolar space drive a renewed and compartmentalised inflammatory response. However, this study was not designed to address this specific question and we cannot exclude the possibility that VAP arises in those patients who first develop an upregulation of inflammation in the lung.

A number of potential biomarkers for VAP have been proposed, including sTREM-1, procalcitonin, copeptin and adrenomedullin. ${ }^{10-12} 22{ }^{23}$ However, only sTREM-1 has been found to have a stronger negative predictive value (or area under the ROC curve) for VAP than the findings presented here,$^{10}$ and recent studies have found less impressive predictive capabilities for sTREM-1 in BALF, ${ }^{24}{ }^{25}$ in line with our observations. It is interesting to consider why IL- $1 \beta$ and IL-8 have not been identified as potential discriminatory markers in previous studies. The compartmentalised nature of proinflammatory cytokines in VAP has been noted previously, ${ }^{26}$ but the diagnostic capability observed in our study was not seen. We believe this discrepancy relates to the timing of investigation and reliance on rigorously collected alveolar samples in our study.

We recommend caution when considering the general applicability of our data. First, our results were generated using bronchoscopy and lavage which may be contraindicated in some patients, and remains relatively labour intensive and operator dependent. ${ }^{27}$ An ideal diagnostic marker for VAP would involve a highly discriminatory blood test. However. this remains elusive, as reflected in Supplementary table S1. Debate continues as to the optimal method for diagnosing VAP. ${ }^{28}$ The "gold standard" for the diagnosis of pneumonia remains histology $y^{29}$; however, this is neither desirable nor practicable in critically ill patients. Therefore, uncertainty over the optimal method of diagnosis affects all studies concerning VAP, especially those focusing on diagnostic markers. A strength of our study lies in the fact that we only recruited patients with predefined clinically suspected VAP using established criteria, ${ }^{1} 17$ and employed a rigorously standardised, visually directed BAL procedure that was conducted by a single experienced operator and relied on quantitative cultures to confirm or refute VAP. To our knowledge these specific, strict criteria have not been simultaneously applied in previous studies seeking diagnostic markers for VAP. Our incidence of confirmed VAP (24\%) is at the lower end of the reported incidence, but is consistent with previous reports, ${ }^{30}$ and the $95 \%$ CI $(14 \%$ to $34 \%)$ for the true population incidence overlaps with the estimate from several other reports. ${ }^{31} 32$ The precise timing of sampling may also influence the diagnosis of VAP. Our data relate specifically to the time of first clinical suspicion of VAP, and are based on the use of a particular assay system. Therefore, while IL-1 $\beta$ and IL-8 are promising diagnostic markers, their diagnostic usefulness (and the derived cut-off values) should be validated in wider populations of ICU patients.

Secondly, there was a non-significant trend towards more "non-VAP" patients being on antibiotics at the time of bronchoscopy. Antibiotics could have suppressed microbial growth to some degree in these patients, although previous studies suggest that after 3 days of unchanged antimicrobial treatment falsenegative cultures are unusual. ${ }^{1}{ }^{13}$ Nevertheless, this does not deflect the fact that marked rises in IL-1 $\beta$ and IL-8 only occurred in patients with $>10^{4} \mathrm{cfu} / \mathrm{ml}$ (figure 3 ). This suggests that low levels of bacterial growth may be tolerated in the alveolar space and support the concept of clinical VAP emerging above a bacterial threshold. ${ }^{115}$

Thirdly, the use of non-ventilated volunteers in this study can be questioned. We would stress that this group was recruited as a reference group only. The important question in clinical practice is whether patients with a high clinical suspicion of VAP have the condition or not; hence our critical comparison was between the VAP and non-VAP groups.

A fourth caveat relates to the variable case mix and microbiological epidemiology of ICUs. ${ }^{33}$ We included a wide variety of surgical and medical cases, but no cardiothoracic cases were involved. The range of bacteria isolated in this study broadly reflects those described elsewhere, but is notable for the absence of Pseudomonas aeruginosa. It may also be potentially relevant that we did not culture for respiratory viruses. Finally, the inclusion of fungi, especially Candida species, as causative organisms in VAP remains controversial. ${ }^{1} 3435$ However, we analysed our data strictly in accordance with our predefined diagnostic definitions 
which were based on the concentration of organisms (without specifying the type). Interestingly we did not find any difference in cytokine levels between fungal and bacterial VAPs, with both demonstrating a profound and characteristic pulmonary inflammation. In our patients with VAP associated with fungal growth, all four cytokines described in figure 1 were above the optimal cut-off levels defined by the ROC curves. While we acknowledge that the small numbers involved mean that statistical non-significance could reflect a type two error, the data suggest that fungal infection was associated with the same pulmonary inflammation as the bacterial pathogens more typically associated with VAP. Furthermore, exclusion of $C$ albicans and/or coagulase-negative staphylococci (sometimes considered "non-pathogenic" organisms) had no significant effect on the diagnostic usefulness of the cytokines as assessed by area under the ROC curve (data not shown).

In conclusion, VAP is associated with increased pulmonary IL-1 $\beta$ and IL-8. Further studies are warranted to validate IL-1 $\beta$ and IL- 8 as diagnostic markers able to influence important clinical endpoints such antibiotic prescribing in ICUs.

Acknowledgements The authors would like to acknowledge the help and support of Ms Pam Ramsay, Mr Gordon Mills and the staff of the Intensive Care Units of the Royal Infirmary of Edinburgh and Western General Hospital, Edinburgh.

Funding Sir Jules Thorn Charitable Trust, 24 Manchester Square, London W1U 3TH. DJD is a Wellcome Trust Career Development Fellow (Fellowship \# 078265). Other Funders: Wellcome Trust.

Competing interests ACM has received an academic prize (travel and accommodation to attend an international conference) funded by Eli Lily. AJS has received expenses from Astra Zeneca and Glaxo Smith Kline (for travel and accommodation) to attend international educational conferences. IFL has received expenses from Astra Zeneca (for travel and accommodation) to attend international educational conferences. TSW is the recipient of an unrestricted educational grant from Wyeth Pharmaceuticals for work concerning epidemiology of ICU-acquired infection. All other authors have no conflicts of interest to declare

Ethics approval This study was conducted with the approval of the Lothian Research Ethics Committee.

ACM collected and analysed data, drafted the manuscript and approves the final version. KK conceived the study, collected and analysed data, drafted the manuscript and approves the final version. TSW collected and analysed data, reviewed the manuscript and approves the final version. OLMN collected and analysed data, reviewed the manuscript and approves the final version. KD collected and analysed data, reviewed the manuscript and approves the final version. LF collected data, reviewed the manuscript and approves the final version. TSW identified patients, assisted in data collection reviewed the manuscript and approves the final version. SJM identified patients, assisted in data collection reviewed the manuscript and approves the final version. DGS identified patients, assisted in data collection reviewed the manuscript and approves the final version. PJA identified patients, assisted in data collection reviewed the manuscript and approves the final version. NA analysed the data, reviewed the manuscript and approves the final version. JRG collected the data, reviewed the manuscript and approves the final version. IFL collected the data, reviewed the manuscript and approves the final version. HR identified the matched volunteers, reviewed the manuscript and approves the final version. DJD analysed the data, reviewed the manuscript and approves the final version. $\mathrm{CH}$ conceived the study, obtained funding, reviewed the manuscript and approves the final version. JMS conceived the study, obtained funding, reviewed the manuscript and approves the final version. AJS conceived the study, obtained funding, collected and analysed the data, drafted the manuscript and approves the final version.

Provenance and peer review Not commissioned; externally peer reviewed.

\section{REFERENCES}

1. Chastre J, Fagon J-Y. Ventilator-associated pneumonia. Am J Respir Crit Care Med 2002:65:867-903

2. Kollef MH. Ventilator-associated pneumonia. A multivariate analysis. JAMA 1993;270:1965-70.

3. Rello J, Ollendorf DA, Oster G, et al. Epidemiology and outcomes of ventilatorassociated pneumonia in a large US database. Chest 2002;122:2115-21.
4. Kollef MH, Morrow LE, Niederman MS, et al. Clinical characteristics and treatment patterns among patients with ventilator-associated pneumonia. Chest 2006;129:1210-18

5. Meduri GU, Mauldin GL, Wunderink RG, et al. Causes of fever and pulmonary densities in patients with clinical manifestations of ventilator-associated pneumonia. Chest 1994:106:221-35.

6. Fagon JY, Chastre J, Wolff M, et al. Invasive and noninvasive strategies for management of suspected ventilator-associated pneumonia. A randomized trial. Ann Intern Med 2000:132:621-3.

7. Conway Morris A, Kefala K, Simpson AJ, et al. Evaluation of diagnostic methodology on the reported incidence of ventilator-associated pneumonia. Thorax 2009;64:516-22

8. Koenig SM, Truwit JD. Ventilator-associated pneumonia: diagnosis, treatment and prevention. Clin Microbiol Rev 2006;19:637-57.

9. Porzecanski I, Bowton DL. Diagnosis and treatment of ventilator-associated pneumonia. Chest 2006;130:597-604.

10. Gibot S, Cravoisy A, Levy B, et al. Soluble TREM-1 and the diagnosis of pneumonia N Engl J Med 2004;350:451-8.

11. Seligman R, Papassotiriou J, Morgenthaler N, et al. Copeptin, a novel prognostic biomarker in ventilator-associated pneumonia. Crit Care 2008:12:R11.

12. Duflo F, Debon R, Monneret G, et al. Alveolar and serum procalcitonin: diagnostic and prognostic value in ventilator associated pneumonia. Anesthesiology 2002;96:74-9.

13. Montravers $\mathbf{P}$, Fagon JY, Chastre J, et al. Follow-up protected specimen brushes to assess treatment in nosocomial pneumonia. Am Rev Respir Dis 1993:147:38-44.

14. Meduri GU, Chastre J. The standardization of bronchoscopic techniques for ventilator-associated pneumonia. Chest 1992;102:557s-63s

15. Baselski VS, Wunderink RG. Bronchoscopic diagnosis of pneumonia. Clin Microbiol Rev 1994:7:533-58.

16. Health Protection Agency. Standard operating procedure, investigation of bronchoalveolar lavage and associated samples Version 5.1. Standards Unit, Evaluation and Standards Laboratory, Specialist and Reference Microbiology Division Health Protection Agency. Issued 2/6/2003.

17. <http://helics.univ-lyon1.fr/>. (accessed $30 \mathrm{Jul} 2009$ )

18. Conway Morris A, Kefala K, Wilkinson TS, et al. C5a mediates peripheral blood neutrophil dysfunction in critically ill patients. Am J Respir Crit Care Med 2009:180:19-28.

19. Rennard SI, Basset G, Lecossier D, et al. Estimation of volume of epithelial lining fuid recovered by lavage using urea as marker of dilution. J Appl Physiol 1986;60:532-8.

20. Youden WJ. An index for rating diagnostic tests. Cancer 1950;3:32-5

21. Donnelly SC, Strieter RM, Kunkel SL, et al. Interleukin-8 and development of adult respiratory distress syndrome in at-risk patient groups. Lancet 1993;341:643-7.

22. Hirata $\mathbf{Y}$, Mitaka $C$, Sato K, et al. Increased circulating adrenomedullin, a novel vasodilatory peptide, in sepsis. J Clin Endocrinol Metab 1996:81:1449-53.

23. Ramirez $\mathbf{P}$, Garcia MA, Ferrer M, et al. Sequential measurements of procalcitonin levels in diagnosing ventilator-associated pneumonia. Eur Respir J 2008;31:356-62

24. Determann RM, Millo JL, Gibot S, et al. Serial changes in soluble triggering recepto expressed on myeloid cells in the lung during development of ventilator associated pneumonia. Intensive Care Med 2005;31:1495-500.

25. Horonenko G, Hoyt JC, Robbins RA, et al. Soluble triggering receptor expressed on myeloid cells-1 is increased in patients with ventilator-associated pneumonia: a preliminary report. Chest 2007;132:58-63.

26. Millo JL, Schultz MJ, Williams C, et al. Compartmentalisation of cytokines and cytokine inhibitors in ventilator-associated pneumonia. Intensive Care Med 2004:30:68-74.

27. Ost D, DeRosiers A, Britt EJ, et al. Assessment of a bronchoscopy simulator. Am J Respir Crit Care Med 2001;64:2248-55.

28. Klompas M. Does this patient have ventilator-associated pneumonia? JAMA 2007;297:1583-93.

29. Rouby JJ, De Lassale EM, Poete P, et al. Nosocomial bronchopneumonia in the critically ill. Histologic and bacteriologic aspects. Am Rev Respir Dis 1992;146:1059-66

30. Tejada Artigas A, Bello Dronda S, Chacón Vallés E, et al. Risk factors for nosocomia pneumonia in critically ill trauma patients. Crit Care Med 2001;29:304-9.

31. Fagon JY, Chastre J, Domart Y, et al. Nosocomial pneumonia in patients receiving continuous mechanical ventilation. Prospective analysis of 52 episodes with use of a protected specimen brush and quantitative culture techniques. Am Rev Respir Dis 1989:139:877-84.

32. Croce MA, Fabian TC, Shaw B, et al. Analysis of charges associated with diagnosis of nosocomial pneumonia: can routine bronchoscopy be justified? J Trauma 1994;37:721-7.

33. Vincent JL, Bihari DJ, Suter M, et al. The prevalence of nosocomial infection in intensive care units in Europe. Results of the European Prevalence of infection in Intensive Care (EPIC) Study. JAMA 1995;274:639-44.

34. Barkauskas CE, Perfect JR. Candida pneumonia:what we know and what we don't Curr Fungal Infect Rep 2009;3:21-31.

35. Azoulay E, Cohen Y, Zahar JR, et al. Practices in non-neutropenic ICU patients with Candida-positive airway specimens. Intensive Care Med 2004;30:1384-9. 


\section{REFERENCES}

1. Anderson SD. Exercise-induced asthma. In: Kay AB, ed. Allergy and allergic diseases. Oxford: Blackwell Scientific Publications, 1997:672-711.

2. Rundell KW, Im J, Mayers LB, et al. Self-reported symptoms and exercise-induced asthma in the elite athlete. Med Sci Sports Exerc 2001; 33:208-13.

3. Dickinson JW, Whyte GP, McConnell AK, et al. Midexpiratory flow versus FEV1 measurements in the diagnosis of exercise induced asthma in elite athletes. Thorax 2006;61:111-4.

4. Holzer K, Douglass JA. Exercise induced bronchoconstriction in elite athletes: measuring the fall. Thorax 2006;61:94-6.

5. Parsons JP, Mastronarde JG. Exercise-induced bronchoconstriction in athletes. Chest 2005;128:3966-74.

\section{Corrections}

doi:10.1136/thx.2009.122291 corr1

Conway Morris A, Kefala K, Wilkinson $\mathrm{TS}$, et al. Diagnostic importance of pulmonary interleukin-1b and interleukin-8 in ventilator-associated pneumonia. Thorax 2010;65:201-7. This article should have included the note that Dr Kefala was joint first author.
Polverino E, Dambrava P, Cilloniz C, et al. Nursing home-acquired pneumonia: a 10 year single-centre experience. Thorax 2010;65:354-59. The correct affiliation for affiliation 1 should have read "Respiratory Department, Hospital Clinic-IDIBAPS, Barcelona-Spain, Centro de Investigación Biomedica En RedEnfermedades Respiratorias (CibeRes, CB06/06/0028, el Ciberes es una iniciativa del ISCIII) - 2009SGRO http://www.idibapsrespiratoryresearch. org."

doi:10.1136/thx.2009.133108corr1

Millett C, Glantz SA. Assigning an '18' rating to movies with tobacco imagery is essential to reduce youth smoking. Thorax 2010;65:377-8. The authors referred to a paper by McNeil et al; this should have been Lyons et al (Lyons A, McNeill A, Chen Y, et al).
Lyons A, McNeill A, Chen Y, et al. Tobacco and tobacco branding in films most popular in the UK from 1989 to 2008. Thorax 2010;65:417-22. There is an error in figure legend 2 which currently reads "Trends in all tobacco intervals and tobacco use intervals per hour per day by British Board of Film Classification (BBFC) category (all figures expressed as means)." It should have read: "Trends in all tobacco intervals and tobacco use intervals per hour per year by British Board of Film Classification (BBFC) category (all figures expressed as means)."

doi:10.1136/thx.2009.127274corr1

Kemp SV, El Batrawy SH, Harrison RN, et al. Learning curves for endobronchial ultrasound using cusum analysis. Thorax 2010;65:534-8. The author name A Roselli should have read A Rosell. 